\section{Rasa LAURINAVICIENE Linda Holm SANDHOLDT Anette BYGUM}

Department of Dermatology and Allergy Centre, Odense University Hospital, DK-5000, Odense C, Denmark

Reprints: R. Laurinaviciene <rlaur@hotmail.dk>

Article accepted on 30/6/2016

\title{
Drug-induced cutaneous lupus erythematosus: 88 new cases
}

\begin{abstract}
Background: An increasing number of drugs have been linked to drug-induced subacute cutaneous lupus erythematosus (DI-SCLE). The recognition and management of DI-SCLE can be challenging, as the condition may be triggered by different classes of drugs after variable lengths of time. Objectives. To determine the proportion of patients with cutaneous lupus erythematosus (CLE) whose drugs are an inducing or aggravating factor. Materials \& methods: We conducted a retrospective chart review of patients diagnosed with CLE at a dermatological department over a 21-year period. We registered clinical, serological, and histological data with a focus on drug intake. Results: Of 775 consecutive patients with a diagnosis of lupus erythematosus (LE) or suspected LE, a diagnosis of CLE could be confirmed in 448 patients. A total of 130 patients had a drug intake that could suggest DI-SCLE. In 88 cases, a drug was evaluated to be definitely, probably, or possibly triggering CLE using the Naranjo probability scale. The most common drugs involved were proton pump inhibitors (PPIs), thiazide diuretics, antifungals, chemotherapeutics, statins, and antiepileptics. The incubation period varied widely with a median of eight weeks. The characteristics of DI-SCLE patients were more widespread rash relative to the other patients, with inflamed skin lesions or atypical variants which could resemble erythema multiforme. Conclusions: We present 88 patients with DI-SCLE, which is the largest case series reported, to date. DISCLE represented $20 \%$ of patients with CLE seen at our department. We conclude that DI-SCLE should be considered in every case of SCLE.
\end{abstract}

Key words: cutaneous lupus erythematosus, drug-induced cutaneous lupus erythematosus, drug reactions
$\mathrm{V}$ arious drugs can induce the development of autoantibodies and clinical features of LE, similar to systemic lupus erythematosus (SLE) or cutaneous lupus erythematosus (CLE). There are currently no standard diagnostic criteria for drug-induced lupus erythematosus (DILE), although it has been defined as a lupus-like syndrome related to continuous drug exposure, which resolves after discontinuation of the drug [1,2]. It has been estimated that $10-15 \%$ of SLE cases are drug induced and that 15,000-30,000 cases of drug-induced systemic lupus erythematosus (DI-SLE) may occur in the United States every year $[1,3-5]$.

The skin manifestations of drug-related CLE are mostly of the SCLE type and symptoms and serological features overlap with those of idiopathic SCLE. Both conditions usually present with annular polycyclic or papulosquamous skin lesions, mostly on sun-exposed areas. However, in contrast to the idiopathic form, the cutaneous manifestations of DISCLE are often more widespread, e.g. with involvement of the lower legs and a tendency to have bullous or erythema multiforme-like skin lesions [2, 6]. Antinuclear antibodies (ANA) and anti-Ro/Sjögren's syndrome A (SSA) antibod- ies are usually present, whereas anti-histone antibodies are rarely found. DI-SCLE can be seen de novo in patients without former CLE or can present as a cutaneous lupus flare in a patient with already known CLE. Incidents of DI-SCLE are unknown. To date, more than 165 cases of DI-SCLE have been published in the English literature [6-26].

A number of different drug categories have been associated with DILE and DI-SCLE, respectively.

The drugs most frequently associated with development of DI-SLE are hydralazine, procainamide, isoniazid, and minocycline [1,5]. DI-SCLE was first reported by Reed et al. in 1985, associated with hydrochlorothiazide [27]. To date, more than 50 drugs have been linked to DI-SCLE, however, the most common triggers are antihypertensive agents, such as thiazide diuretics, calcium channel blockers and angiotensin-converting enzyme inhibitors, terbinafine, proton pump inhibitors (PPIs), lipid lowering agents, or chemotherapeutic agents $[6,26,28]$. Over the past decade, an increasing number of drugs in different classes have been recognised as triggers for DILE and the number is constantly increasing, in proportion to the licensing of new medications on the market, such as TNF- $\alpha$ antagonists. 
Drug-induced chronic cutaneous lupus erythematosus (CCLE), which is rarely reported in the literature, usually refers to fluorouracil, non-steroidal anti-inflammatory drugs, and TNF- $\alpha$ antagonists, such as infliximab and adalimumab $[1,5,29]$.

The diagnosis and management of DI-SCLE is usually simple when the condition is recognised and the patient is taking only a single drug known to be a trigger for DI-SCLE. In patients on multiple and essential drugs, the situation can be quite challenging.

The aim of this study was to determine the proportion of patients with CLE whose drugs were an inducing or aggravating factor, and to compare our findings with the current literature.

\section{Material and Methods}

\section{Study design}

A retrospective chart review was conducted in patients with CLE seen at the Department of Dermatology and Allergy Centre, Odense University Hospital, Denmark, between January 1994 and December 2014. Odense University Hospital covers the region of southern Denmark, which has 1.2 million inhabitants.

\section{Identifying cases}

Inclusion criteria were the following ICD-10 diagnoses: M32.0: drug-induced systemic lupus erythematosus; M32.1: systemic lupus erythematosus with organ or system involvement; M32.2: other forms of systemic lupus erythematosus; M32.9: systemic lupus erythematosus, nonspecified; L93.0: discoid lupus erythematosus (CCLE), lupus erythematosus not otherwise specified; L93.1: subacute cutaneous lupus erythematosus; and L93.2: other local lupus erythematosus, lupus erythematosus profundus (lupus panniculitis), and lupus erythematosus tumidus.

Medical records were reviewed to identify possible DISCLE cases.

Since July 2007, all patients with CLE were also registered using the European Society of Cutaneous Lupus Erythematosus (EUSCLE) Core Set Questionnaire, leading to the identification of any sign or suspicion of DILE.

\section{Collection of data}

Medical records from patients using potential lupustriggering medications were analysed. In several cases, it was necessary to request additional information from general practitioners, other departments, or from the personal electronic medicine profile collected in relation to their clinical course. The medical records were reviewed for clinical course, treatment, and histopathological findings. The following data were obtained from the hospital records: patient gender, age at first patient contact in relation to current rash, previous history of skin symptoms, latency and resolution period, medication information, objective findings, serological and histopathological data, as well as therapeutic measures.

\section{Causality assessment}

We used the Naranjo algorithm to estimate the causal relationship between drugs and cutaneous reactions in the identified cases [30]. This scoring system consists of ten questions, answered as "yes", "no", or "do not know", resulting in a score to each question ranging from -1 to +2 . Based on the total score, the adverse drug reaction (ADR) is divided into the following probability categories: definite, probable, possible, or doubtful. Patients with doubtful ADRs were excluded from this study and only patients with definite, probable, or possible causality were analysed.

\section{Ethics}

Permission was obtained from the Danish Data Protection Agency, J.nr 2012-41-0927.

\section{Results}

A total of 775 medical records were reviewed and we identified 448 patients with CLE. A total of 130 of the 448 patients had a drug intake that could suggest drug-related CLE. Forty-two cases were excluded as doubtful and/or due to insufficient data based on the medical records. In 88 cases, the drug history was evaluated to be definitely, probably, or possibly related to CLE, and their clinical data are presented in Annexe Atable S1. A summary of the characteristics of the 88 patients with DI-SCLE are presented in table 1. The remaining LE patients without drug-related factors were classified as idiopathic cases.

Fifty patients exhibited de novo DI-SCLE and 38 patients had a previous history of CLE exacerbated by LE-triggering drugs. With the probability score, we demonstrated that five of the 88 patients had a definite, 53 had a probable, and 30 had a possible drug/CLE relationship. The median age at the initial visit was 63 years (range: 28-91 years; mean: 61 years). In total, $86 \%$ of the patients were female.

\section{Drugs implicated in DI-CLE}

In the group of patients with definite/probable/possible DICLE, 11 different drugs were implicated (figure 1). The

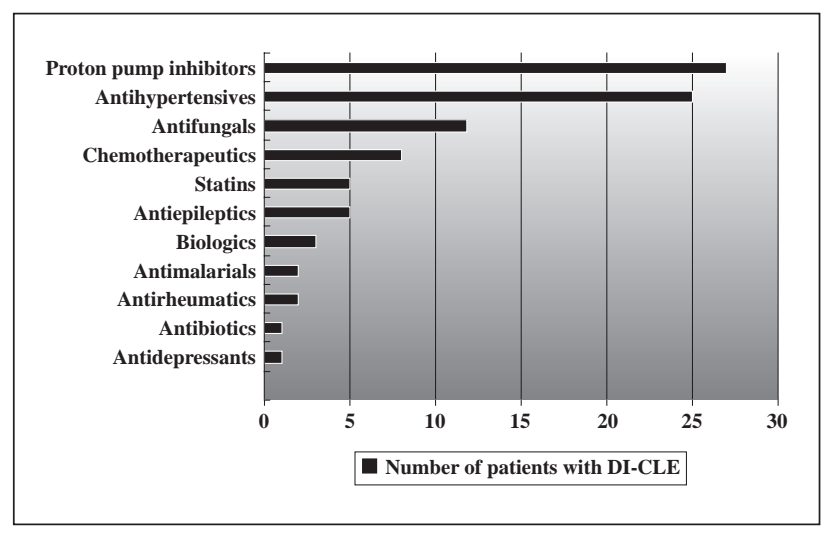

Figure 1. Eighty-eight cases of drug-induced cutaneous lupus erythematosus (DI-CLE) precipitated by 11 different classes of medications. 
Table 1. Characteristics of 88 patients with drug-induced cutaneous lupus erythematosus (DI-CLE).

\begin{tabular}{|c|c|c|c|c|c|c|c|c|}
\hline Sex & Age (years) & $\begin{array}{l}\text { Type of } \\
\text { DI-CLE }\end{array}$ & $\begin{array}{l}\text { Incubation } \\
\text { period }\end{array}$ & $\begin{array}{l}\text { Autoantibodies } \\
\text { in relation to } \\
\text { DI-CLE }\end{array}$ & Histopathology & Treatment & $\begin{array}{l}\text { Resolution } \\
\text { period }\end{array}$ & $\begin{array}{l}\text { Naranjo } \\
\text { probability } \\
\text { score [30] }\end{array}$ \\
\hline $\begin{array}{l}12 \text { males } \\
(14 \%)\end{array}$ & $28-91$ & $\begin{array}{l}\text { De novo } \\
\text { DI-SCLE } \\
n=50(57 \%)\end{array}$ & $\begin{array}{l}\text { Median: } \\
8 \text { weeks }\end{array}$ & $\begin{array}{l}\text { Positive ANA } \\
54 / 85 \text { tested } \\
\text { patients }(64 \%)\end{array}$ & $\begin{array}{l}\text { Confirmed CLE: } \\
83 / 83 \text { skin } \\
\text { biopsies }(100 \%)\end{array}$ & $\begin{array}{l}\text { Topical } \\
\text { corticosteroids } \\
n=76(86 \%)\end{array}$ & $\begin{array}{l}\text { Median: } \\
9 \text { weeks }\end{array}$ & $\begin{array}{l}\text { Definite: } \\
n=5(6 \%)\end{array}$ \\
\hline \multirow[t]{4}{*}{$\begin{array}{l}76 \text { females } \\
(86 \%)\end{array}$} & Median:63 & $\begin{array}{l}\text { Previous } \\
\text { DI-SCLE } \\
n=38(43 \%)\end{array}$ & & $\begin{array}{l}\text { Positive SSA } \\
45 / 61 \text { tested } \\
\text { patients }(74 \%)\end{array}$ & $\begin{array}{l}\text { Positive DIF: } \\
21 / 66 \text { skin } \\
\text { biopsies } \\
(32 \%)\end{array}$ & $\begin{array}{l}\text { Systemic therapies } \\
\text { (hydroxychloro- } \\
\text { quine, prednisolone, } \\
\text { methotrexate, }\end{array}$ & & $\begin{array}{l}\text { Probable: } \\
n=53 \\
(60 \%)\end{array}$ \\
\hline & Mean:61 & & & $\begin{array}{l}\text { Positive SSB } \\
13 / 52 \text { tested } \\
\text { patients }(25 \%)\end{array}$ & & $\begin{array}{l}\text { azathioprine, } \\
\text { isotretinoin, } \\
\text { mycophenolate }\end{array}$ & & $\begin{array}{l}\text { Possible: } \\
n=30 \\
(34 \%)\end{array}$ \\
\hline & & & & $\begin{array}{l}\text { Positive dsDNA } \\
10 / 47 \text { tested } \\
\text { patients }(21 \%)\end{array}$ & & $\begin{array}{l}\text { mofetil) } \\
n=51(58 \%)\end{array}$ & & \\
\hline & & & & $\begin{array}{l}\text { Positive histone } \\
8 / 46 \text { tested } \\
\text { patients }(17 \%)\end{array}$ & & & & \\
\hline
\end{tabular}

CLE: cutaneous lupus erythematosus; DI-CLE: drug-induced cutaneous lupus erythematosus; SCLE: subacute cutaneous lupus erythematosus; incubation period: delay from prescription of drug to onset of rash; DIF: direct immunofluorescence; ANA: antinuclear antibodies; SSA: anti-Ro/SSA antibodies; SSB: anti-La/ SSB antibodies; dsDNA: antibodies to double-stranded DNA; histone: anti-histone antibodies.

most common culprit drugs included PPIs $(n=27)$, thiazide diuretics or combinations thereof $(n=21)$, antifungals $(n=12)$, chemotherapeutic agents $(n=8)$, statins $(n=5)$, and antiepileptics $(n=5)$.

In the first decade of our study, there was a clear predominance of antihypertensives, almost exclusively thiazide diuretics, as culprit drugs, but currently PPI-induced DISCLE predominate (figure 2).

The median incubation time for all drug classes was eight weeks (range: three days to 10 years; mean: 54 weeks). In 78 cases, the suspected drug was stopped and the skin rash disappeared after a median of nine weeks (range: one week to one year; mean: 13 weeks). In seven patients, a causal relation between CLE and medication was not noticed,

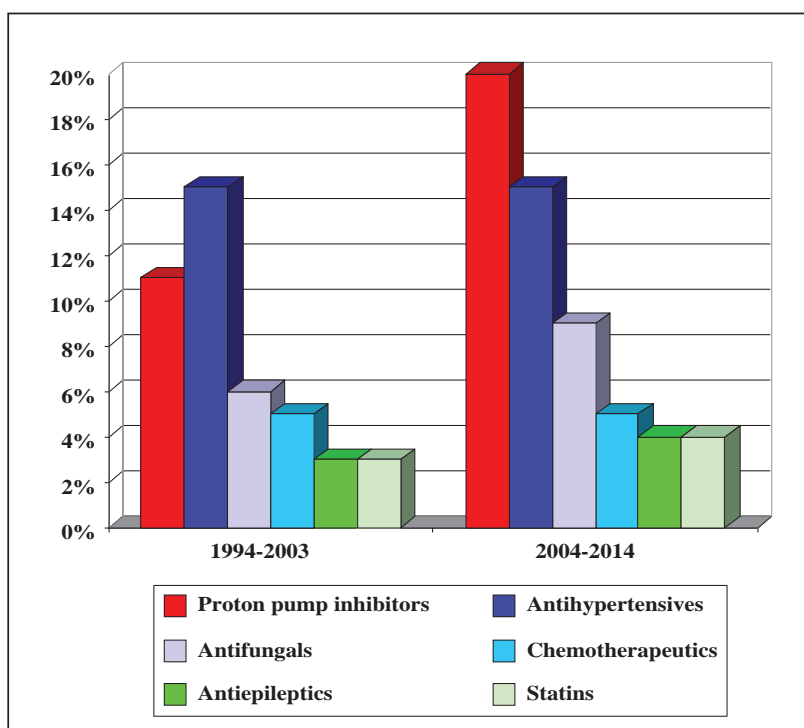

Figure 2. Proportion of drugs precipitating cutaneous lupus in the first and second decade of our study period. and the consumption of drug continued. In three patients, it was not considered possible to substitute the culprit drug.

\section{Treatment}

Most patients received topical treatment with corticosteroids ( $86 \%$ of patients). Of the patients, $36 \%$ received additional hydroxychloroquine and $31 \%$ additional prednisolone. Three patients $(3.4 \%)$ received methotrexate, three patients azathioprine, one patient (1\%) isotretinoin, and one patient additional mycophenolate mofetil. Of these patients, $18 \%$ received a combination of two or three different systemic medications and $48 \%$ were treated with a combination of topical and systemic therapy.

\section{Relapsing DI-SCLE}

Eleven of our patients had multiple episodes of DI-SCLE and three experienced a lupus rash provoked by different pharmacological agents. Two of them had reactions to an antifungal and PPI and one reacted to PPI and a thiazide diuretic. Four patients experienced lupus rash induced by two different PPIs and two patients had SCLE, twice induced by hydrochlorothiazide-containing antihypertensives. Two patients had three episodes induced by agents within the same pharmacological group: PPIs (pantoprazole, esomeprazole) and chemotherapeutics (epirubicin, docetaxel).

\section{Characteristics of DI-CLE}

In 16 of 88 patients (18\%) with DI-CLE, the skin rash was widespread, with involvement of the lower extremities. Bullous or erythema multiforme-like/toxic epidermal necrolysis-like lesions were seen in 18 patients $(20 \%)$ and vasculitic lesions were observed in two patients $(2 \%)$ (figure 3).

Immunological examination at the time of the rash showed positive ANA in 54 of 85 tested patients (64\%), 


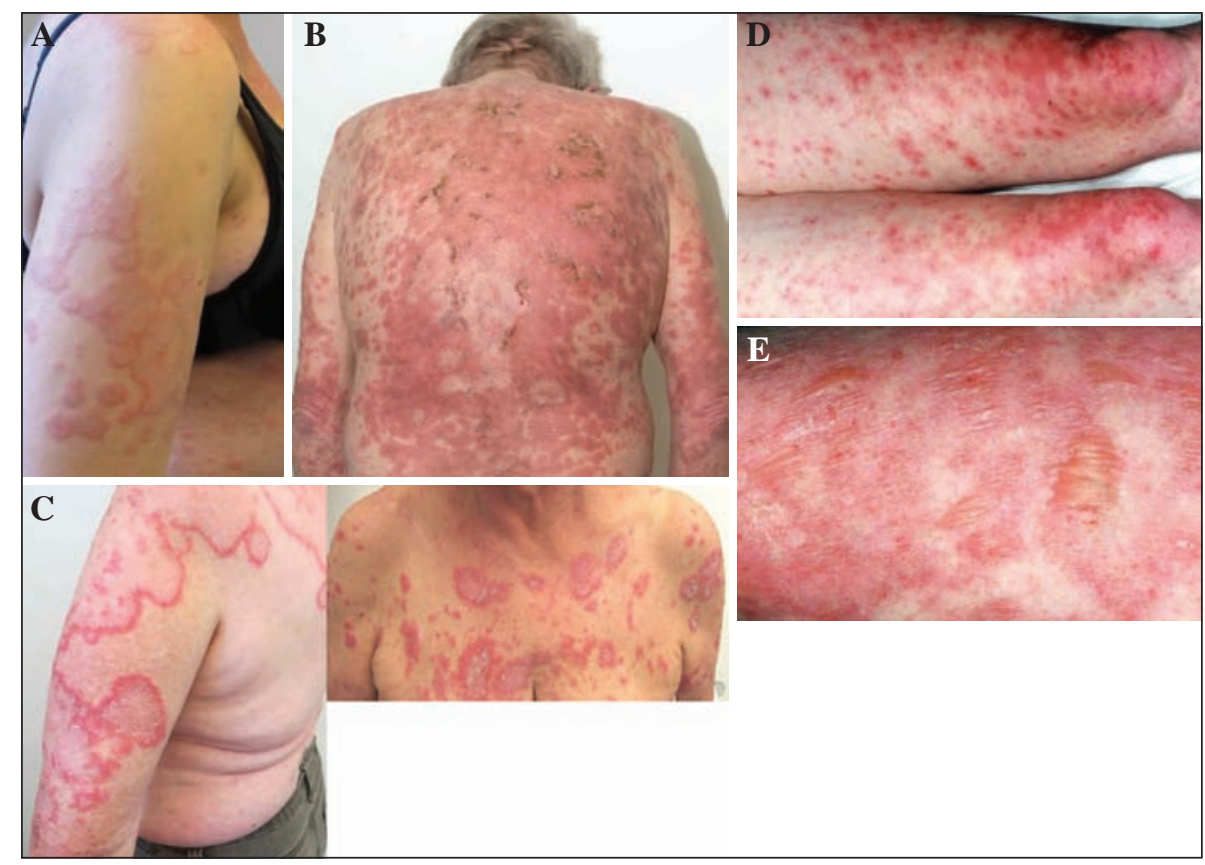

Figure 3. Patients with drug-induced subacute cutaneous lupus erythematosus. A) Erythema multiforme-like lesions; culprit drug was lansoprazole. B) Erosive skin rash with haemorrhagic crusts; culprit drug was terbinafine. C) (left panel) Annular and polycyclic subacute cutaneous lupus erythematosus lesions; culprit drug was omeprazole. C) (right panel) Papulosquamous subacute cutaneous lupus erythematosus lesions; culprit drug was terbinafine. D) Widespread skin rash with involvement of the lower extremities; culprit drug was terbinafine. E) Bullous, toxic epidermal necrolysis-like skin eruption; culprit drug was terbinafine.

anti-Ro/SSA antibodies in 45 of 61 tested patients $(74 \%)$, anti-La/ SSB antibodies in 13 of 52 tested patients $(25 \%)$, antibodies to double-stranded DNA in 10 of 47 tested patients $(21 \%)$, and anti-histone antibodies were detected in 8 of 46 tested patients $(17 \%)$ at the time of the skin eruption. Positive direct immunofluorescence (DIF) was seen in 21 of 66 performed skin biopsies (32\%).

The characteristics of DI-SCLE are presented in table 2.

\section{Discussion}

Traditionally, DILE is believed to be an SLE-like condition with multiorgan involvement, including myoarthralgias, serositis, fever, weight loss and sometimes rash accompanied by anti-histone antibodies, and a lack of lupus specific antibodies [4, 31]. Dermatologists usually see a different clinical picture dominated by skin manifestations, accompanied by Ro/SSA-antibodies.

Our study is the largest published series of consecutive lupus patients seen in a single dermatological department. In accordance with our clinical impression, we found a substantial proportion of CLE patients to have DI-CLE (20\%), mostly of the SCLE type. It was previously estimated that about $6-20 \%$ of SCLE are drug-related $[32,33]$, but recent studies have suggested that the incidence of drug-induced SCLE may be even higher [26, 34]. Grönhagen found that $71 \%$ of patients with incident SCLE had been exposed to a drug previously implicated in DI-SCLE during the six months prior to the diagnosis of SCLE [26]. This means that DI-SCLE is not uncommon, although it may go unnoticed by the patient and treating physician, assuming it to be idiopathic SCLE.

Table 2. Characteristics of DI-SCLE.

\begin{tabular}{|ll|}
\hline Age of onset & Older \\
\hline Female/male & $9: 1$ \\
\hline Clinical course & $\begin{array}{l}\text { Related to drug exposure, median incubation time: } 8 \text { weeks. } \\
\text { Resolving after drug withdrawal, median resolution time: } 9 \text { weeks. }\end{array}$ \\
\hline The most likely culprit drugs & PPIs, antihypertensives, antifungals. \\
\hline Clinical features & $\begin{array}{l}\text { Annular or papulosquamous lesions on sun-exposed skin. } \\
\text { The widespread presentation and occurrence of bullous, targetoid and vasculitic } \\
\text { manifestations. } \\
\text { Involvement of the lower legs is highly suspicious of DI-SCLE. }\end{array}$ \\
\hline Histopathological characteristics & Similar to idiopathic SCLE. \\
\hline Immunological characteristics & $\begin{array}{l}\text { Similar to idiopathic SCLE. } \\
\text { Anti-Ro/SSA, ANA antibodies are usually present. }\end{array}$ \\
\hline
\end{tabular}


Based on our data, PPIs and thiazide diuretics were the most common inducing/aggravating drugs. Antihypertensive agents were sometimes combined with a thiazide, which further complicated the interpretation of the culprit drug. The culprit drugs relate to the prescription pattern in the individual countries and possible drug changes over time. Interestingly, in the first decade of our study, we saw a preponderance of thiazide diuretics as eliciting drugs, but currently PPI-induced DI-SCLE predominate (figure 2) [6]. PPIs are one of the most commonly prescribed drugs and prescription of PPI in Denmark has increased three-fold during the last 10 years [6]. According to our data, other inducing/aggravating drugs are antifungals, chemotherapeutics, statins, and antiepileptics. In a population-based case-control study of 234 patients with SCLE from Sweden, a significantly increased risk of SCLE was found after exposure to terbinafine, TNF- $\alpha$ inhibitors, antiepileptics, PPIs, thrombocyte inhibitors, ACE inhibitors, and NSAIDs [26]. Surprisingly, thiazide diuretics were not mentioned. Eleven of our patients had multiple episodes of lupus rash and six of them experienced lupus rash induced by different medications within the same pharmacological group. This phenomenon was noticed for different PPIs and chemotherapeutics. Three of our patients had SCLE episodes induced or aggravated by two different pharmacological groups. The patients with drug-aggravated LE had a previous history of CLE and Sjögren syndrome. It seems that patients with earlier CLE and known autoimmune diseases, especially Sjögren syndrome, can be prone to develop drug-induced or exacerbated SCLE in response to certain culprit drug classes [5]. Cross-reactivity between different PPIs has been discussed in our previous report [6]. This should lead to special precautions and information regarding future prescriptions for patients with early DI-CLE, as they have a risk of recurrence. Three of our patients had SCLE induced by etanercept. Whether skin reactions to TNF- $\alpha$ inhibitors should be classified separately has been a topic for discussion, as the pathomechanism may differ. We chose deliberately, and in accordance with Grönhagen et al. [26], to include these cases in our study.

Most cases of DI-CLE develop several weeks, months, and sometimes years from the initial prescription, and the manifestations will usually disappear weeks to months after drug intake has been stopped.

The delay in our patient cohort was from three days to 10 years, with a median of eight weeks, which is longer than that reported from a systematic review reporting that the incubation period of DI-SCLE ranged from three days to 11 years (average: 27.9 weeks; median: six weeks) [26, 31]. We found that antiepileptics and antihypertensives (mostly thiazide diuretics) had the longest incubation period, with a median of two years (range: two weeks to 10 years; average: 3.2 years for antiepileptics, and range: two weeks to eight years; average: 2.4 years for antihypertensives). Chemotherapeutic agents, hydroxychloroquine and tetracycline, had a shorter incubation period, with a median of 1-2 weeks.

The resolution period of the lupus rash after stopping the culprit drugs ranged from two weeks to 1.5 years, with an average of 13 weeks (median: nine weeks). In this period, patients received treatment with topical corticosteroids and most also received systemic therapies, such as hydroxychloroquine, prednisolone, or other immunosuppressive drugs.
No pathognomonic clinical or paraclinical features can, with any certainty, be used to distinguish between DI-SCLE and idiopathic SCLE at the moment. All biopsied patients (83 of 88) had a diagnosis of CLE which was confirmed histologically, however, our pathologists could not distinguish between DI-SCLE and idiopathic SCLE. Also, direct immunofluorescense findings did not differ in patients with idiopathic SCLE. However bullous, erosive or toxic epidermal necrolysis-like findings were found in $20 \%$ of the patients, indicating a more inflammatory picture. Also, concomitant vasculitic lesions were found in a few patients. The skin rash may also be more widespread and inflamed, and it has been reported that involvement of the lower legs is highly suspicious of drug-related SCLE [2]. In this and our previous study of PPI-induced DI-SCLE, we also noticed a tendency for more widespread and erosive, sometimes erythema multiforme-like, lesions in patients with DI-SCLE, as opposed to idiopathic SCLE [6].

In general, $>80 \%$ patients with DI-SCLE are ANA positive, and about $80 \%$ of patients with drug-induced SCLE are also positive for Ro/SSA, while positivity for $\mathrm{La} / \mathrm{SSB}$ is seen in $<50 \%$ of patients and anti-histone antibodies in $33 \%$ [31]. In our study, immunological examination showed positive ANA in 64\%, anti-Ro/SSA antibodies in 74\%, anti-La/ SSB antibodies in $25 \%$, antibodies to double-stranded DNA in $21 \%$, and anti-histone antibodies were detected in $17 \%$ at the time of the rash. Four of eight patients with positive antihistone antibodies had a diagnosis of SLE, but DI-SLE was not identified in any of the patients. Autoantibodies usually disappear much slower than clinical symptoms. According to a US study, antibody titres decreased in only $40 \%$ of patients after eight months of follow-up and remained detectable at significant titres for less than two years in another groups of patients [4]. In our study, serological data were measured at the time patients presented with DI-CLE, while we only sporadically made follow-up measurements after the disappearance of the clinical signs, and therefore reliable data on this issue could not be collected in this retrospective study.

The limitations of this study are its retrospective nature and small number of subjects with definite DI-SCLE. A definite relationship with drug intake was identified in only five patients, as the Naranjo scoring requires that: (1) a definite reaction should follow a reasonable temporal sequence following drug intake; (2) there is a recognised response to the suspected drug; (3) the reaction improves on withdrawal of the drug; and (4) the reaction reappears on re-exposure. Re-exposure and drug provocation have seldom been performed in this group of patients, because it is not ethically feasible to introduce re-exposure, bearing in mind that this may provoke a severe drug rash sometimes with erythema multiforme or toxic epidermal necrolysis-like skin eruption. Based on our data, accidental re-exposure of the eliciting drugs was noticed in eight patients who were unintentionally prescribed the culprit drug. Seven patients developed the same type of skin reaction, while one patient seemingly tolerated the drug the second time.

A possible relationship with drugs was not necessarily a focus for the physician who saw the patient during the acute phase. In cases where drug information was not detailed, a report by the general practitioner and files from other departments were sought, to make sure that no possible drug associations were overlooked. We found examples of patients being followed in the outpatient clinic for up to 
10 years, before an inducing/aggravating drug was stopped and the rash cleared. We also noticed patients hospitalised with an LE rash who were discharged from our ward with a thiazide diuretic. Later, a suspicion of DI-SCLE was raised and the drug stopped, leading to clearance of the eruption. In the daily clinic, it is our impression that a high proportion of patients with LE flare or newly diagnosed SCLE are treated with potentially "lupus-precipitating" drugs. In such cases, it is very important to recognise the drug association, as skin rash will usually disappear when the eliciting drug is stopped. More intensive pharmacological treatment should be reserved for resistant cases, which usually represent a pre-existing LE exacerbated by the offending drug. Identification and discontinuation of the implicated medication is the cornerstone of DI-SCLE treatment.

Disclosure. Financial support: none. Conflict of interest: none.

\section{References}

1. Vedove CD, Del Giglio M, Schena D, Girolomoni G. Drug-induced lupus erythematosus. Arch Dermatol Res 2009; 301:99-105.

2. Marzano AV, Lazzari R, Polloni I, Crosti C, Fabbri $P$, Cugno $M$. Drug-induced subacute cutaneous lupus erythematosus: evidence for differences from its idiopathic counterpart. $\mathrm{Br} J$ Dermatol 201 1; 165: 335-41.

3. Lorentz K, Booken N, Goerdt S, Goebeler M. Subacute cutaneous lupus erythematosus induced by terbinafine: case report and review of literature. J Dtsch Dermatol Ges 2008; 6: 823-7.

4. Borchers AT, Keen CL, Gershwin ME. Drug-induced lupus. Ann N Y Acad Sci 2007; 1 108: 166-82.

5. Pretel M, Marquès L, España A. Drug-induced lupus erythematosus. Actas Dermosifiliogr 2014; 105: 18-30.

6. Sandholdt LH, Laurinaviciene R, Bygum A. Proton pump inhibitorinduced subacute cutaneous lupus erythematosus. $\mathrm{Br} J$ Dermatol 2014; 170: 342-51

7. Sifuentes Giraldo WA, Ahijón Lana M, García Villanueva M, González García C, Vázquez Diaz M. Chilblain lupus induced by TNF- $\alpha$ antagonists: a case report and literature review. Clin Rheumatol 2012; 31:563-8

8. Andric $M$, Dixit $S$, Robaei $D$, Watchorn $R$, Verma N. A case of subacute cutaneous lupus erythematosus as a result of ranibizumab (Lucentis) treatment. Indian J Ophthalmol 2013;61:752-4.

9. Lamond NW, Younis T, Purdy K, Dorreen MS. Drug-induced subacute cutaneous lupus erythematosus associated with nab-paclitaxel therapy. Curr Oncol 2013; 20: 484-7.

10. Buchanan S, Rosemergy I, Healy P. Drug-induced subacute cutaneous lupus erythematosus due to treatment with interferon beta-1 a. $N$ Z Med J 2013; 126: 98-101

11. Cleaver N, Ramirez J, Gildenberg S. Cutaneous lupus erythematosus in a patient undergoing intravitreal bevacizumab injections: case report and review of the literature. J Drugs Dermatol 2013; 12: 1052-5.

12. Wiznia LE, Subtil $A$, Choi JN. Subacute cutaneous lupus erythematosus induced by chemotherapy: gemcitabine as a causative agent. JAMA Dermatol 2013; 149: 1071-5.

13. Yildirim Cetin G, Sayar H, Ozkan F, Kurtulus S, Kesici F, Sayarlioglu M. A case of toxic epidermal necrolysis-like skin lesions with systemic lupus erythematosus and review of the literature. Lupus 2013; 22: 839-46.

14. Almebayadh $M$, Regnier-Rosencher $E$, Carlotti $A$, et al. Subacute cutaneous lupus erythematosus induced and exacerbated by proton pump inhibitors. Dermatology 2013;226: $119-23$.
15. Lis-Święty A, Brzezińska-Wcisło L, Widuchowska M, Kucharz E. Subacute cutaneous lupus erythematosus in the course of rheumatoid arthritis: a relationship with TNF- $\alpha$ antagonists and rituximab therapy? Immunopharmacol Immunotoxicol 2013;35: 443-6.

16. Chen JK, Chen TS, Lim $P$, labal M. Drug-induced subacute cutaneous lupus erythematosus associated with doxorubicin. J Am Acad Dermatol 2012; 67: 273-5.

17. Wilkerson E, Hazey MA, Bahrami S, Callen JP. Golimumabexacerbated subacute cutaneous lupus erythematosus. Arch Dermatol 2012; 148: $1186-90$

18. Cabanillas $M$, Suárez-Amor $O$, Ramírez-Santos $A$, et al. Lamotrigine induced subacute cutaneous lupus erythematous. Dermatol Online J 2012; 18: 12 .

19. Sakai T, Hatano $Y$, Abe I, Ishii K, Fujiwara S. A case of an SLE patient with febrile neutropenia who experienced exacerbation of cutaneous manifestations after the administration of G-CSF. Mod Rheumatol 2013; 23: 1231-6.

20. Marchetti MA, Noland MM, Dillon PM, Greer KE. Taxane associated subacute cutaneous lupus erythematosus. Dermatol Online J 2013; 19: 19259.

21. Wong NY, Parsons $L M$, Trotter M, Tsang RY. Drug-induced subacute cutaneous lupus erythematosus associated with docetaxel chemotherapy: a case report. BMC Res Notes 2014; 7: 785.

22. Kwong HL, Teo RY. An uncommon cutaneous reaction for a common drug. Diagnosis: drug-induced subacute cutaneous lupus erythematosus (DI-SCLE). Ann Acad Med Singapore 2014; 43: 433-4.

23. Reinholz $M$, Berking $C$, Hermans $C$, Ruzicka $T$, Braun-Falco $M$. Lupus erythematosus-like skin eruption after vemurafenib therapy. J Am Acad Dermatol 2014; 71: 159-60.

24. Liakou Al, Brunner M, Theodorakis M, Makrantonaki E, Zouboulis CC. Recurrent subacute cutaneous lupus erythematosus following exposure to different drugs. Acta Derm Venereol 201 1;91:586-7.

25. Murie J, Agarwal M. Sub-acute cutaneous lupus erythematosus following nitrofurantoin: causative or coincidental? Scott Med J 2014; 59:21-5.

26. Grönhagen $C M$, Fored $C M$, Linder $M$, Granath F, Nyberg F. Subacute cutaneous lupus erythematosus and its association with drugs: a population-based matched case-control study of 234 patients in Sweden. Br J Dermatol 2012; 167: 296-305

27. Reed BR, Huff JC, Jones SK, Orton PW, Lee LA, Norris DA. Subacute cutaneous lupus erythematosus associated with hydrochlorothiazide therapy. Ann Intern Med 1985; 103: 49-51.

28. Callen JP. Drug-induced subacute cutaneous lupus erythematosus. Lupus 2010; 19: $1107-11$.

29. Vedove CD, Simon JC, Girolomoni G. Drug-induced lupus erythematosus with emphasis on skin manifestations and the role of anti-TNF $\alpha$ agents. J Dtsch Dermatol Ges 2012; 10: 889-97.

30. Naranjo $C A$, Busto $U$, Sellers $E M$, et al. A method for estimating the probability of adverse drug reactions. Clin Pharmacol Ther 1981; 30: 239-45.

31. Lowe G, Henderson CL, Grau RH, Hansen CB, Sontheimer RD. A systematic review of drug induced subacute cutaneous lupus erythematosus. Br J Dermatol 201 1; 164: 465-72.

32. Biazar $C$, Sigges J, Patsinakidis $N$, et al. Cutaneous lupus erythematosus: first multicenter database analysis of 1,002 patients from the European Society of Cutaneous Lupus Erythematosus (EUSCLE). Autoimmun Rev 2013; 12: 444-54.

33. Vasoo S. Drug-induced lupus: an update. Lupus 2006; 15 : 757-61

34. Callen JP. Consider drugs as a cause or an exacerbating factor in patients diagnosed with subacute cutaneous lupus erythematosus! $\mathrm{Br} J$ Dermatol 2012; 167: 227-8.

\section{Supplementary data}

Supplementary data associated with this article can be found, in the online version, at doi:10.1684/ejd.2016.2912. 